\title{
Relaxation effects on the magnetism of decorated step edges: $\mathrm{Co} / \mathrm{Pt}(664)$
}

\author{
S. Baud and Ch. Ramseyer \\ Laboratoire de physique moléculaire, UMR CNRS 6624, Université de Franche-Comté, F-25030 Besançon Cedex, France \\ G. Bihlmayer* and S. Blügel \\ Institut für Festkörperforschung, Forschungszentrum Jülich, D-52425 Jülich, Germany \\ (Received 1 February 2005; revised manuscript received 25 January 2006; published 20 March 2006)
}

\begin{abstract}
Employing first-principles calculations, we investigate the relaxations of a clean and Co decorated Pt stepedge on a $\langle 111\rangle$ microfaceted (111) surface. The visibility of the relaxation in scanning tunneling microscopy (STM) images is discussed. We calculate the magnetocrystalline anisotropy energy (MAE) of the Co decorated vicinal surface with and without relaxation effects taken into account and compare our results to experiments done for $\mathrm{Co} / \mathrm{Pt}(997)$ and to previous calculations with different geometries simulating the vicinal surface. As compared to the unrelaxed case, the relaxation of the Co chain towards the substrate enhances the contribution of Pt to the MAE and turns the easy axis more in the direction of the surface plane.
\end{abstract}

DOI: 10.1103/PhysRevB.73.104427

PACS number(s): 75.75.+a, 75.30.Gw, 73.20.-r

\section{INTRODUCTION}

Both on the experimental and on the theoretical side, the field of low-dimensional magnetism has attracted considerable interest: due to the lower coordination, magnetic moments are usually larger in thin films or wires than in the bulk and several elements that are nonmagnetic as bulk materials become magnetic in low dimensions. ${ }^{1}$ A large increase of the spin magnetic moment was observed when going from bulk systems to thin films. Continuing the reduction of dimensionality to the ultimate limit, recently several studies reported on an enormous increase of the orbital moments in atomic scale systems. ${ }^{2-4}$ These large orbital moments are accompanied by considerable magnetic anisotropies that were found experimentally.

$A b$ initio calculations based on density functional theory in the local density approximation (LDA) or the generalized gradient approximation have, rather successfully, described two-dimensional magnetic systems. ${ }^{5-7}$ Although it might seem surprising at first sight that a theory developed for a dilute, homogeneous electron gas is successful in describing this rather inhomogeneous situation, several studies show that it is possible to obtain both the magnetic anisotropy and-with the inclusion of some correction terms to the LDA - the orbital moments reasonably well. ${ }^{3,8,9}$ Since in low-dimensional systems the coordination of the atoms is low, relaxations that try to compensate for this loss of coordination can be expected to be large. Up until now, most computational studies employed simplified structural models and the effect of relaxations was not taken into account. The underlying physics, i.e., the reduction of the coordination of the magnetic atom and the low symmetry of the system, is already contained in these models. Therefore, when applied to magnetic chains on stepped surfaces, they successfully describe the characteristic magnetic properties, e.g., the rather large magnetic anisotropy and the unusual direction of the easy axis in these systems. ${ }^{8,9}$ Apart from these more qualitative aspects, it is worthwhile to investigate the quantitative changes that result from improved structural models. Since the structural rearrangements that occur in these sys- tems have the effect to strengthen the hybridization between the atoms, it can be expected that this tends to counteract the increase in magnetic moments and magnetic anisotropy that has been computed in the unrelaxed structures.

Motivated by pioneering experiments, ${ }^{2,4}$ we have chosen a Co chain deposited on a Pt step edge to investigate the effect of a precise structural modeling. From the mismatch of the Pt and Co lattice parameters (almost 10\%) and the natural relaxations at a step edge, we can expect substantial effects here. After a short description of the computational method and our structural model in Sec. II, we describe the results of our structural optimization (both for a clean and a Co-decorated Pt step edge) and compare it to the experimental data in Sec. III. Employing this structure, we then present the orbital moments and magnetocrystalline anisotropy energies of the relaxed and unrelaxed Co wire on the Pt surface in Sec. IV.

\section{STRUCTURAL MODEL AND COMPUTATIONAL METHOD}

To simulate the stepped surfaces, we calculated vicinal $\mathrm{Pt}(111)$ surfaces of a terrace width of 6 atomic rows. On a fcc (111) surface, two types of close packed steps can be formed: the A-type step, where the step-edge atoms and the surface atoms of the lower terrace form a square unit cell [like on a fcc (100) surface], and the B-type step, where these atoms form a triangle, as on a fcc (111) surface. Therefore, these two types of steps are called $\langle 100\rangle$ microfaceted or $\langle 111\rangle$ microfaceted, respectively. Vicinal surfaces can be regarded as periodic arrays of a single type of step edges separated by terraces. For example, a $(p+1, p-1, p-1)$ surface consists of terraces with a width of $p$ full atomic rows and terminated by A-type steps, in $(p, p, p-2)$ surfaces the terraces terminate with B-type steps. Therefore, we calculated thin films of $\mathrm{Pt}(755)$ and (664) surfaces, including in total 46 and 45 atoms, respectively. For the simulation of the decorated steps, the Pt edge atoms were substituted by Co.

The calculations were performed by the $a b$ initio full- 
TABLE I. Relaxations (in percent of the ideal bulk value) of the nearest-neighbor bond length of the step-edge atom on clean, stepped $\operatorname{Pt}(111)$ surfaces. The positions of the nearest-neighbor $\mathrm{Pt}$ atoms are indicated in Fig. 1. As a model we used a 26 layer Pt(755) film for the A-type step and a 45 layer Pt(664) film for the B-type step. The results are compared to Ref. 15 (BLS), where (644) and (442) surfaces were considered, and to Ref. 16 (F), where (533) and (442) surfaces were used to model the A- and B-type steps, respectively. In the last line the step formation energy (SFE) for the relaxed surfaces calculated in GGA are given.

\begin{tabular}{lcccccc}
\hline \hline & \multicolumn{3}{c}{ A-type step } & \multicolumn{3}{c}{ B-type step } \\
\cline { 2 - 7 } Bond direction & This & BLS & F & This & BLS & F \\
\hline On upper terrace & -3.1 & -2.2 & -2.5 & -3.3 & -3.2 & -3.0 \\
Into bulk & -3.0 & -3.6 & -3.2 & -4.8 & -4.8 & -4.9 \\
To lower terrace & -0.1 & -1.3 & -2.4 & -1.7 & -2.4 & -2.4 \\
SFE (eV/atom) & 0.28 & 0.29 & & 0.24 & 0.25 & \\
\hline \hline
\end{tabular}

potential linearized augmented plane wave $(\text { FLAPW })^{10}$ method, as implemented in the FLEUR code. ${ }^{11}$ The calculations are based on the generalized gradient approximation ${ }^{12}$ to the (spin) density functional theory. Spin-orbit coupling was included, as described in Ref. 13. For all structures the calculated $\mathrm{Pt}(111)$ in-plane lattice constant $(2.81 \AA)$ was used, and the six topmost interlayer distances were relaxed by force calculations. ${ }^{14}$ For these calculations approximately 80 basis functions/atom and $5 \mathbf{k}_{\|}$points in the irreducible part of the surface Brillouin zone (2D-BZ) have been used. In the simulation of the STM images, this number was increased to $40 \mathbf{k}_{\|}$points. The magnetocrystalline anisotropy energy (MCA) was calculated applying the force theorem, starting from a self-consistent calculation, where spin-orbit coupling was included. The MCA was evaluated using $128 \mathbf{k}_{\|}$points in the full 2D-BZ. The dependence of the calculated quantities on the number of $\mathrm{k}$ points was tested: the calculated energies were found to be stable to within $0.3 \mathrm{meV} / \mathrm{atom}$, the direction of the easy axis did not change by more than $5^{\circ}$, and the orbital moment was almost unaffected. The applicability of the force theorem was checked for certain configurations by comparison with self-consistent calculations. An average deviation of $0.2 \mathrm{meV} /$ atom between the different methods was observed.

The magnetic anisotropy energy (MAE) is a sum of the MCA and the shape anisotropy. The latter favors a magnetization direction parallel to the wire. Since its magnitude is in the order of $0.1 \mathrm{meV}$ and, therefore, much smaller than the MCA, we will not consider the shape anisotropy in the following.

\section{RELAXATION AND SIMULATION OF STM IMAGES}

\section{A. Clean Pt(664) surface}

Let us first discuss clean, stepped Pt(111) surfaces with Aand B-type steps. Relaxations of these types of step edges were determined by both Boisvert et al. ${ }^{15}$ and Feibelman. ${ }^{16}$ Both calculations show inward relaxations of the step atoms of $3 \%-4 \%$ (as compared to the unrelaxed, ideal bulk interatomic distances; cf. Table I). Although the surfaces that were used in these references differ in terrace width (4 or 5 atomic rows per terrace), a general agreement between these results and our calculation is obvious. Also the calculated step formation energies and their ratio (0.88) is in good agreement with the existing GGA calculation ${ }^{15}$ and experimental data. ${ }^{17}$

From the comparisons above, we can be quite confident that our calculated relaxations are reliable. Note that it is difficult to compare with experimental results of the stepped surface. Indeed, one can find some x-ray diffraction measurements and simulations used to study the structure of $\mathrm{Pt}(997),{ }^{18}$ but these results are mostly qualitative and do not allow for a quantitative comparison. Essentially the authors in Ref. 18 underline the fact that to understand and interpret correctly the experimental x-ray spectra, one has to consider a collective displacement of atoms near the steps and not only the atoms constituting the step. That is exactly what we did since several layers were allowed to relax. In general, it is known that the (111) surfaces of fcc metals do not relax much, since the (111) face is already quite densely packed. In particular, for the platinum substrate, it is evident from both experimental and theoretical studies, that the surface layer exhibits a small outward relaxation $\left(+1.3 \%{ }^{19}\right.$ and $\left.+1.1 \%{ }^{20}\right)$. Here, in the case of the $\operatorname{Pt}(664)$ surface, this tendency is also observed for the atoms constituting the terraces that show a small outward relaxation. Among these atoms, only the step edge atom shows an inward relaxation, leading to the decrease of the distances to its nearest neighbor atoms on the upper (Pt-u) and lower (Pt-l) terrace and into the bulk (Pt-b), as presented in Table I. It is the low coordination of the step edge atom that causes the relaxation toward the bulk in order to increase its electron density and therefore lower the surface energy.

This calculated inward relaxation seems to contradict experimentally observed STM images of this step edge. ${ }^{21}$ Indeed, what can be observed on various experimental topography images is an outward relaxation of the STM tip near the step edge. But this may be of electronic origin, e.g., the presence of a localized surface (or step) state along the step edge pointing toward the STM tip could be at the origin of such an observation. We have tried to reproduce theoretically the STM images of the Pt step edge. According to the model of Tersoff and Hamann ${ }^{22}$ to describe the tunneling current 


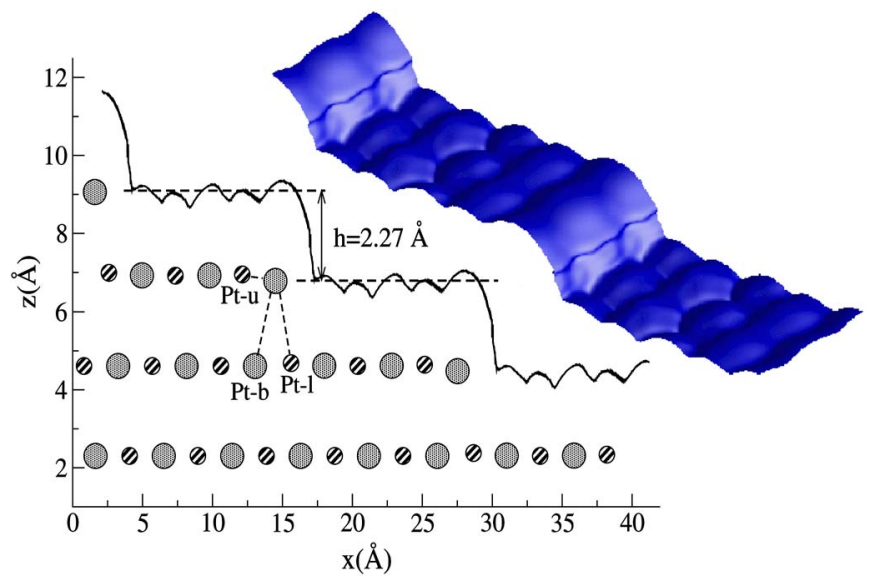

FIG. 1. (Color online) Simulation of a STM image of the clean Pt step-edge at $-10 \mathrm{meV}$ tunneling voltage. A cross section through the relaxed step and a simulated line scan normal to the step edge (in the $x z$ plane, including the step-edge atom) is shown on the left. Atoms within the same $x z$ plane are drawn in the same size. The three Pt atoms that are nearest neighbors to the step-edge atom are indicated: in the upper terrace (Pt-u), the lower terrace (Pt-1) and in the direction of the bulk (Pt-b). On the upper right an isosurface of the local DOS in the vacuum from the occupied states with less than $10 \mathrm{meV}$ binding energy is shown.

measured in STM, the variation of the tunneling current $I$ with respect to the bias voltage $U$ is proportional to the local density of states $\rho$ of the sample in the vacuum at the tip position $\vec{r}$, i.e., $d I / d U \propto \rho\left(\vec{r}, E_{F}+e U\right)$, where $E_{F}$ is the Fermi energy. Thus, the energy integrated local density of states of occupied $(U<0)$ or unoccupied $(U>0)$ states in the energy range $\left[E_{F}, E_{F}+e U\right]$ contribute to the tunneling current, $I(\vec{r})$ $\propto \int_{E_{F}}^{E_{F}+e U} \rho(\vec{r}, E) d E$. This model was successfully applied for bias voltages of $\pm 2 \mathrm{eV}^{23}$

Referring to the STM images obtained by Gambardella et $a l .,{ }^{21}$ we find that they are generally obtained for bias volt- ages of the order of about $-10 \mathrm{meV}$. To obtain images comparable to the experimental ones, the local density of states was integrated over the energy range $\left[E_{F}-10 \mathrm{meV} ; E_{F}\right]$. In order to simulate a constant current image $\bar{\rho}(\vec{r})$ $=\int_{E_{F}-10 \mathrm{meV}}^{E_{F}} \rho(\vec{r}, E) d E$ was evaluated for a number of positions $z$ of the tip in the vacuum, and the positions $\{\vec{r}\}$ were plotted for a grid of in-plane $(x, y)$ values and a given constant value of $\bar{\rho}$.

Figure 1 shows a simulated line scan normal to the step edge and the isosurface of the local DOS in the vacuum. It can be observed that the image obtained by theory is quite similar to the experimental ones that are generally found in the literature: it is possible to identify the hexagonal structure of the terraces as well as the different rows in the terrace. From the line scan, it is also possible to extract the average apparent height difference between two successive terraces. The so determined value of $2.27 \AA$ is in agreement with the experimental value of $2.35 \pm 0.05 \AA$. But, finally, neither a clear enhancement of the isodensity or an increase of the tip-sample distance at constant current, respectively, near the step edge, nor an inward relaxation can be observed in the simulated STM images. We have also checked that no surface state was present along the step edge by studying the band structure of the relaxed $\mathrm{Pt}(644)$ surface. Figure 2 presents the band structure along the $(\bar{\Gamma} \bar{Y} \bar{S} \bar{X} \bar{\Gamma})$ direction. States localized more than $50 \%$ in the terrace atoms are marked by (red) squares. From this picture, one can see that it is possible to identify two surface states around an energy of $0.3 \mathrm{eV}$ above $E_{F}$ along the directions $(\overline{\mathrm{Y}} \overline{\mathrm{S}})$ and $(\overline{\mathrm{X}} \bar{\Gamma})$, which are perpendicular to the step edge. Although it is unlikely that these states contribute to the observed increase of the tip height near the step edge, we checked that images obtained by integrating $\rho$ up to $E_{F}+0.3 \mathrm{eV}$ did not differ from the one presented here, in the sense that no clear enhancement of the isodensity appeared. Either the experimentally observed outward relaxation is due to an electronic effect that is specific to the Pt(997) surface (like a quantum well state that depends

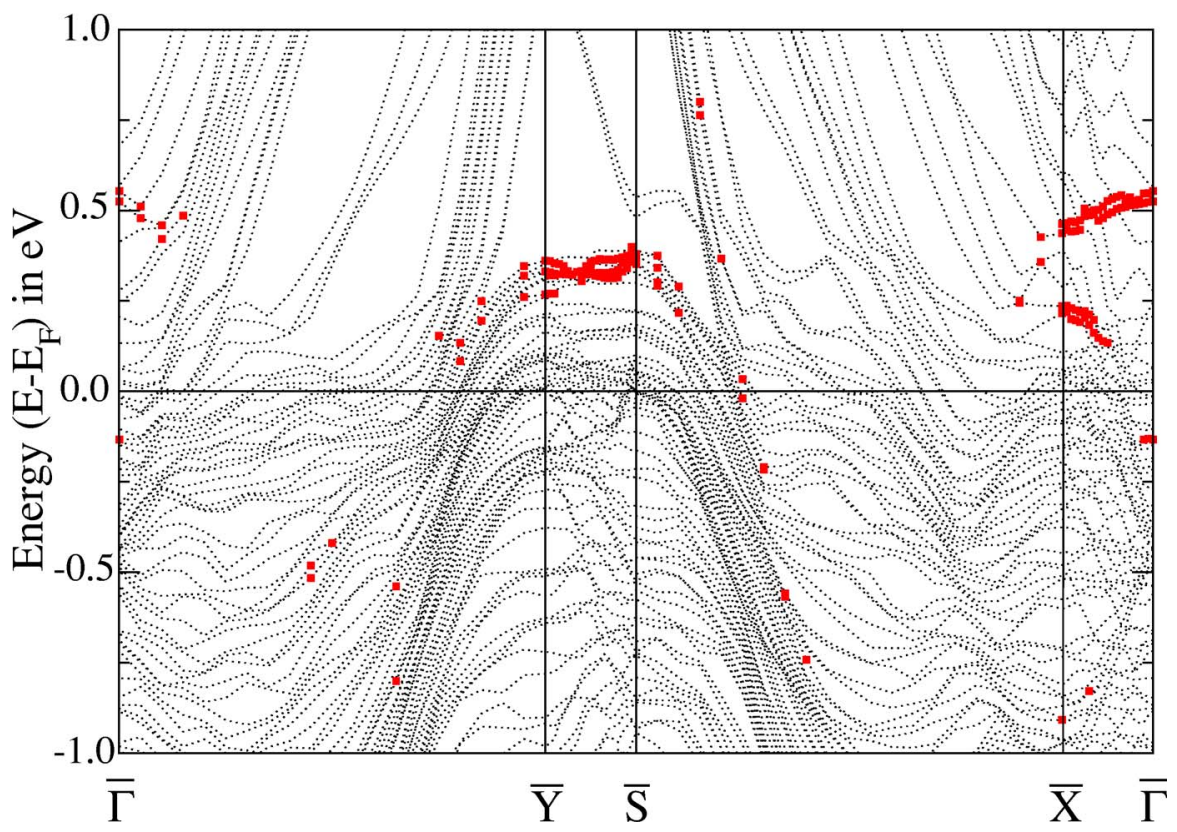

FIG. 2. (Color online) Band structure of the $\operatorname{Pt}(664)$ vicinal surface. Squares (red) mark the states whose localization on the terrace atoms is over $50 \%$. 


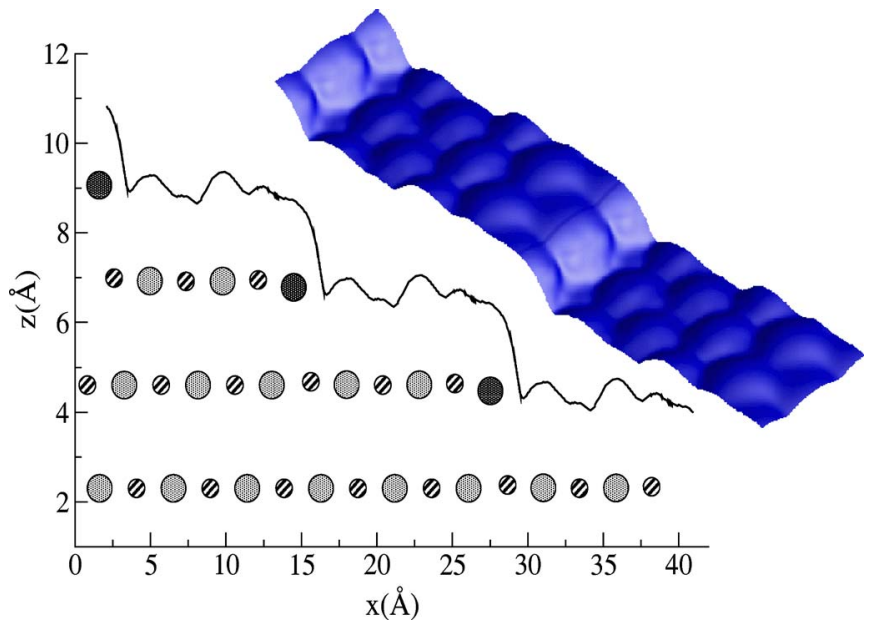

FIG. 3. (Color online) The same as Fig. 1, but for the Co decorated step edge. The Co atoms are shown as dark filled circles.

sensitively on the terrace width) or our applied model to simulate STM images is too simple to show this effect. Calculations with more realistic models of the STM tip or experiments using different tips could provide information to separate effects of the tip from properties of the sample. Our model also did not include any tip-sample interaction that might occur at low bias voltages. STM topographs recorded at different tunneling voltages could help to resolve this question.

\section{B. Co decorated Pt step edge}

The relaxations of $\mathrm{Co}$ atoms in a chain decorating the B-type Pt(111) step edge are larger than for the clean $\mathrm{Pt}(664)$ surface, e.g., the distance of the Co atom to the next Pt atom in the terrace changes by $-7.3 \%$, the distance to the next $\mathrm{Pt}$ atom on the lower terrace by $-7.4 \%$, and the distance to the nearest atom in the bulk, even by $-13.3 \%$; all values are given with respect to the ideal atom spacing in bulk Pt. As in the case of the clean Pt substrate, we simulated STM images in the presence of the Co chain along the step edge. The simulated line scan normal to the step and the isosurface of constant local density of states is presented in Fig. 3. They were obtained with the same energy integration range $\left[E_{F}\right.$ $\left.-10 \mathrm{meV} ; E_{F}\right]$ as the one used previously for the $\operatorname{Pt}(664)$ system. Here, contrary to the bare platinum vicinal surface, the line scan is in agreement with the inward relaxation of the Co atom. Even if it is not so clear in the 3D plot, it is possible to see that the Co atoms forming the step edge are imaged a little bit lower than the Pt atoms forming the terraces. On the other hand, it appears clearly that along the step edge the image presents more corrugation, thus allowing us to identify each individual Co atom of the adsorbed chain. This feature was not observed for the vicinal Pt surface. This possible identification of the Co atom with this remarkable corrugation is also found in the experimental STM images. ${ }^{4}$

\section{ORBITAL AND MAGNETIC ANISOTROPY}

Before we study now the magnetic properties of the $\mathrm{Pt}$ deposited Co wire, we first recapitulate the experimental re- sults and the results of two recent theoretical studies on this system. Experimentally, ${ }^{4}$ the easy axis of the magnetization was determined to be perpendicular to chain direction, pointing $43^{\circ}$ off the terrace normal, in the direction of the upper terrace. From the magnetization curves at a temperature of $45 \mathrm{~K}$ an anisotropy energy of $2.0 \pm 0.2 \mathrm{meV}$ per atom was determined and the orbital moment of Co was found to be $0.68 \mu_{\mathrm{B}}$. The orbital moment anisotropy of Co was reported in Ref. 4 to be $0.12 \mu_{\mathrm{B}}$. Shick and co-workers ${ }^{8}$ simulated the $\operatorname{Pt}(997)$ surface by a $p(6 \times 1)$ unit cell of two layers of $\mathrm{Pt}(111)$, where three rows of $\mathrm{Pt}$ and one row of Co were deposited. These three Pt rows then simulate the upper terrace, while the remaining two (empty) rows open up the lower terrace. Újfalussy et al. ${ }^{9}$ put a chain of seven Co atoms in a missing double-row of $\mathrm{Pt}$ atoms in a $\mathrm{Pt}(111)$ surface. The cluster of the $\mathrm{Co}$ and all neighboring $\mathrm{Pt}$ atoms were then embedded in a $\mathrm{Pt}(111)$ surface. In both calculations no relaxations were taken into account, i.e., the Co atoms sit at the (ideal) Pt position. Both calculations find an easy axis that is perpendicular to the chain direction, pointing $18^{\circ}$ (Ref. 8) and $42^{\circ}$ (Ref. 9) away from the terrace normal, in remarkable agreement with the experimental value. Apart from this obvious success, other quantities differ considerably: In Ref. 8 the magnetocrystalline anisotropy energy difference between the hard and easy axis is $4.45 \mathrm{meV}$, the terrace-normal $(z)$ direction is about $3 \mathrm{meV}$ more favorable than the in-chain direction, and the orbital moment in the $z$ direction is $0.15 \mu_{\mathrm{B}}$. In Ref. 9 the hard and easy axis differ by $1.42 \mathrm{meV}$, now the $z$ direction is about $0.28 \mathrm{meV}$ less favorable than the in-chain direction, and the orbital moments are between 0.19 and $0.20 \mu_{\mathrm{B}}$.

\section{A. Unrelaxed $\mathrm{Co} / \operatorname{Pt}(664)$}

First, we consider the MAE for different directions of the spin-quantization axis for the unrelaxed configuration. From the left panel of Fig. 4 we can see, that also in our calculation the easy axis is tilted similar to the experimental and earlier mentioned theoretical results, this time the angle with the terrace normal is $51^{\circ}$. This is in fine agreement with the experiment, as is the energy difference of $2.5 \mathrm{meV}$ between the hard and easy axes.

Problems arise when we look at the orbital moments: from Fig. 5 we see, that the orbital moment is about $0.14 \mu_{\mathrm{B}}$ for the $z$ direction, and the orbital moment anisotropy is only about $0.03 \mu_{\mathrm{B}}$. Shick et al. ${ }^{8}$ used the LDA $+\mathrm{U}$ method to improve the orbital moments, that are normally underestimated in LDA or GGA. This now favorably increased the orbital moment to $0.45 \mu_{\mathrm{B}}$ [a calculation ${ }^{24}$ using the orbital polarization (OP) correction even led to a value of $0.92 \mu_{\mathrm{B}}$ ], but nobody so far calculated the MAE for wires in schemes beyond LDA. Earlier attempts to enhance the orbital moments of adatoms on noble metal surfaces with the OP correction $^{25}$ were accompanied also by an increase of the MAE and led to extremely high anisotropies [about $70 \mathrm{meV}$ for $\mathrm{Co}$ on $\mathrm{Ag}(001)]$. When an OP correction would be applied to $\mathrm{Co} / \mathrm{Pt}(664)$, so that the OMA agrees with the experimentally observed value of $0.12 \mu_{\mathrm{B}}$, a similar enhancement of the MAE could be expected. ${ }^{26}$ The magnitude of this en- 


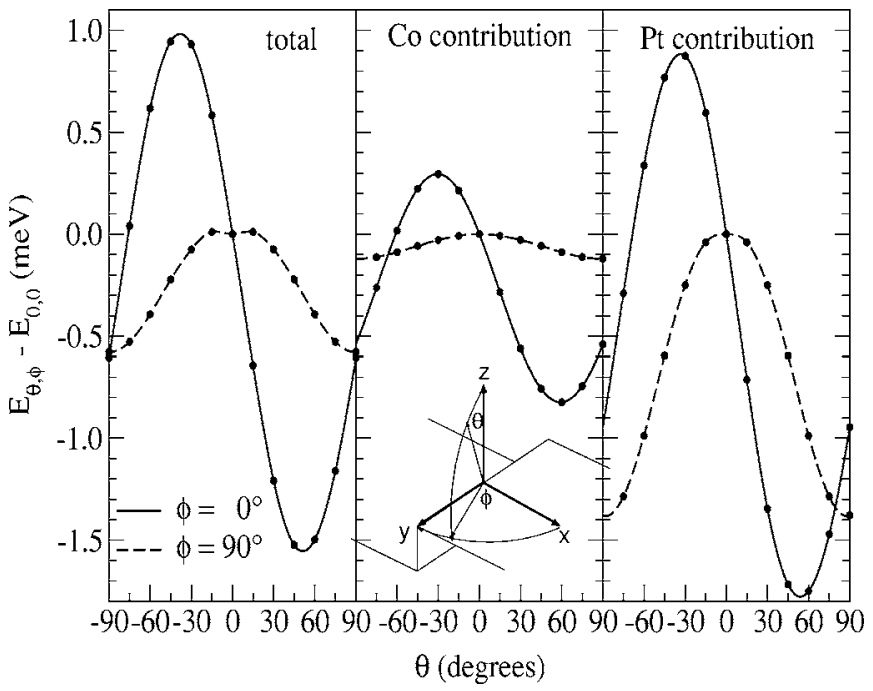

FIG. 4. Energy of the unrelaxed Co decorated Pt step edge as a function of the spin-quantization axis (SQA) calculated with the force theorem. Contributions of the Co and a nearby $\mathrm{Pt}$ atom are shown in the middle and right panels, respectively. These contributions have been evaluated by selectively including spin-orbit coupling only in the spheres of the Co or the Pt atoms, respectively. Full lines correspond to a variation of the SQA in the $x z$ plane, dashed lines correspond to a variation in the $y z$ plane.

hancement will depend on the contribution of the Co chain to the MAE. How large this contribution is compared to that of the substrate will be investigated in this section. When comparing to the experimentally reported MAE, one has to keep in mind that the measurements of Ref. 4 were actually performed on magnetized samples above their Curie temperature. It is therefore a priori not clear how well this value should correspond to the MAE obtained from a ground-state calculation.

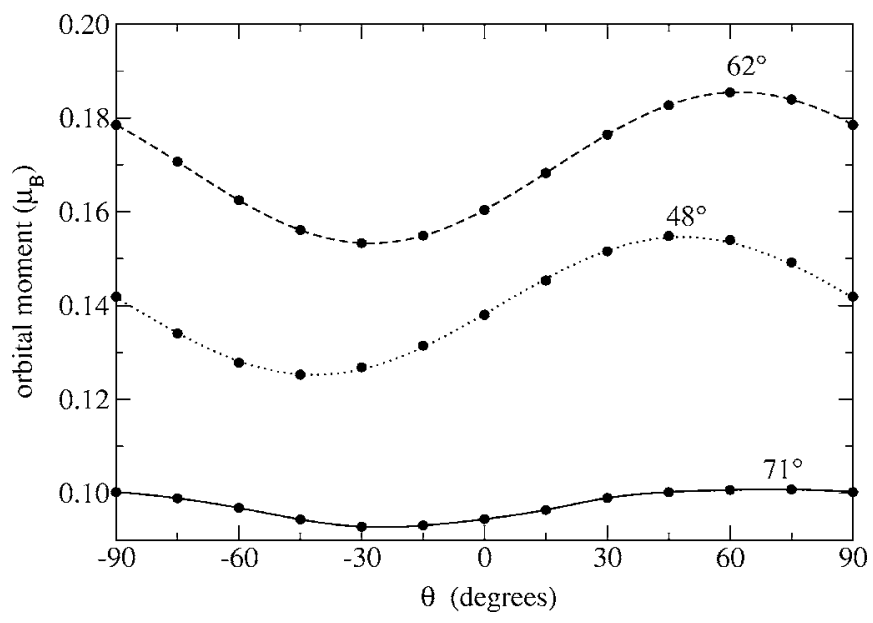

FIG. 5. Orbital moments of the Co atom at the step edge as a function of the SQA rotated in a plane perpendicular to the step edge. Shown are the results for the relaxed (full line) and unrelaxed (dotted line) structures, as well as for a calculation of the relaxed structure, where spin-orbit coupling was only taken into account at the Co site (dashed line). The positions of the maxima are indicated by the numbers in the plot.
An important observation in this context is that the total MAE observed in this system consists, not only of the contributions of the Co chain, but also includes substantial contributions of the Pt substrate that is polarized by the magnetic wire. Studying Fe and Co alloys, Solovyev and co-workers ${ }^{27}$ already realized the implications of alloying a magnetic transition metal atom with heavier elements for the MAE. Similar observations have been made for small Co clusters on $\mathrm{Pt}(111) .{ }^{3}$ The importance of the contributions of the neighboring $\mathrm{Pt}$ atoms for the direction of the easy axis in Co chains on Pt step edges was discussed in Ref. 8, where it was shown that the occurrence of an easy axis that is tilted with respect to the terrace normal is a consequence of the different contributions of the nearest neighbor Pt-Co pairs to the anisotropy (i.e., the difference between the atoms marked as Pt-b and Pt-l in Fig. 1). In Table II we have listed the spin and orbital magnetic moments of the cobalt atom and the three nearest neighbor Pt atoms as well as the sum of all $\mathrm{Pt}$ moments in (one side of) the film. From this table we find, that in the unrelaxed film the induced Pt moments are quite large and, in total, the induced spin moment of $\mathrm{Pt}$ is about $50 \%$ of the Co moment. The percentage is even larger, when we look at the induced orbital moments, which are in total larger in $\mathrm{Pt}$ than in Co. The total orbital moment anisotropy (OMA) of the Pt atoms is of the same order of magnitude as the OMA of $\mathrm{Co}$, although the directions of the magnetizations, which leads to the strongest orbital moments, are different for the Co and Pt contributions.

These calculations were performed self-consistently, i.e., without using the force theorem, for several magnetization directions. The agreement between the values given by the force theorem, and the self-consistent results for the orbital moments is very good. We also checked the energy difference between easy and hard axes in the structurally relaxed and unrelaxed system by comparing self-consistent calculations to the results of the force theorem and found that the magnetocrystalline anisotropies are overestimated by the latter method by $4 \%$ in the relaxed and $13 \%$ in the unrelaxed case.

To illustrate the relative importance of Co and Pt contributions to the MAE is to perform calculations using the force theorem, but setting the spin-orbit coupling constants of either Co or the Pt atoms to zero. Such a decomposition is shown in the right part of Fig. 4. We see that the MAE from the $\mathrm{Pt}$ atoms is more than two times larger than the MAE stemming from the Co atoms. One has to note here that the sum of Co and Pt contributions to the MAE is not simply the total MAE of the system, since the presence or absence of the spin-orbit coupling term in one part of the atoms also influences the shape of the wave functions in the other part of the system. It is also interesting to notice here that the OMA found in Table II is well reflected in Fig. 4: Of the two in-plane directions $\left(\theta=90^{\circ}\right)$, the Pt contribution favors the in-chain direction $\left(\phi=90^{\circ}\right)$, while the Co atom prefers the perpendicular $\left(\phi=0^{\circ}\right)$ direction.

\section{B. Relaxed Co/Pt(664)}

Let us now investigate the influence of the relaxation on the orbital moment of Co, the MAE and the easy axis: As can 
TABLE II. Spin $\left(\mu_{S}\right)$ and orbital moments $\left(\mu_{L}\right)$ for the atoms in the unrelaxed and relaxed Co/Pt(664) structures. The orbital moments for the spin-quantization axis perpendicular to the vicinal surface $(\theta, \phi=0,0)$, parallel to the surface, but perpendicular to the Co wire ( $\theta, \phi=\pi / 2,0)$, and parallel to the Co wire $(\theta, \phi=\pi / 2, \pi / 2)$ is given. The moments for the Pt atoms nearest to the Co atom in the upper terrace (marked as Pt-u in Fig. 1), in the bulk (Pt-b) and on the lower terrace (Pt-1) are given, as well as the sum of all Pt moments in the film $(\Sigma \mathrm{Pt})$.

\begin{tabular}{|c|c|c|c|c|c|c|c|c|}
\hline \multirow[b]{2}{*}{ Atom } & \multicolumn{4}{|c|}{ Unrelaxed } & \multicolumn{4}{|c|}{ Relaxed } \\
\hline & $\mu_{S}$ & 0,0 & $\begin{array}{c}\mu_{L} \\
\frac{\pi}{2}, 0\end{array}$ & $\frac{\pi}{2}, \frac{\pi}{2}$ & $\mu_{S}$ & 0,0 & $\begin{array}{c}\mu_{L} \\
\frac{\pi}{2}, 0\end{array}$ & $\frac{\pi}{2}, \frac{\pi}{2}$ \\
\hline Co & 2.148 & 0.133 & 0.147 & 0.123 & 2.105 & 0.093 & 0.101 & 0.060 \\
\hline Pt-u & 0.174 & 0.035 & 0.043 & 0.056 & 0.239 & 0.047 & 0.066 & 0.083 \\
\hline Pt-b & 0.148 & 0.027 & 0.031 & 0.035 & 0.220 & 0.045 & 0.044 & 0.054 \\
\hline Pt-1 & 0.170 & 0.030 & 0.039 & 0.035 & 0.231 & 0.047 & 0.059 & 0.064 \\
\hline$\Sigma \mathrm{Pt}$ & 1.087 & 0.173 & 0.188 & 0.191 & 1.168 & 0.210 & 0.240 & 0.257 \\
\hline
\end{tabular}

be inferred from Fig. 5, the orbital moment of Co and its anisotropy are drastically reduced (the orbital moment of Co in the relaxed structure is $30 \%$ smaller than in the unrelaxed one, the OMA is reduced by a factor of 3.7). From Table II it can be seen that the reduction of the Co spin and orbital moments is compensated by an increase of the induced $\mathrm{Pt}$ moments. The hybridization of Co with the substrate increases with the relaxation, thereby increasing the effective crystal field splitting at the Co sites and reducing their orbital moment. But at the same time, the closer vicinity of Co and $\mathrm{Pt}$ also results now in larger induced moments at the Pt atoms. The total induced orbital moment at the Pt sites is then more than twice as large as the Co orbital moment.

The value of MAE is almost unchanged by the relaxation (around $2.2 \mathrm{meV}$, cf. the left panel of Fig. 6), at least if only the hard and easy axis are compared. Looking at the decomposition of the MAE into Co and Pt contributions (the middle and right panels of Fig. 6), we observe a similar trend as for the orbital moments: the Co contribution to the MAE is reduced, while the $\mathrm{Pt}$ dominates the contribution to the

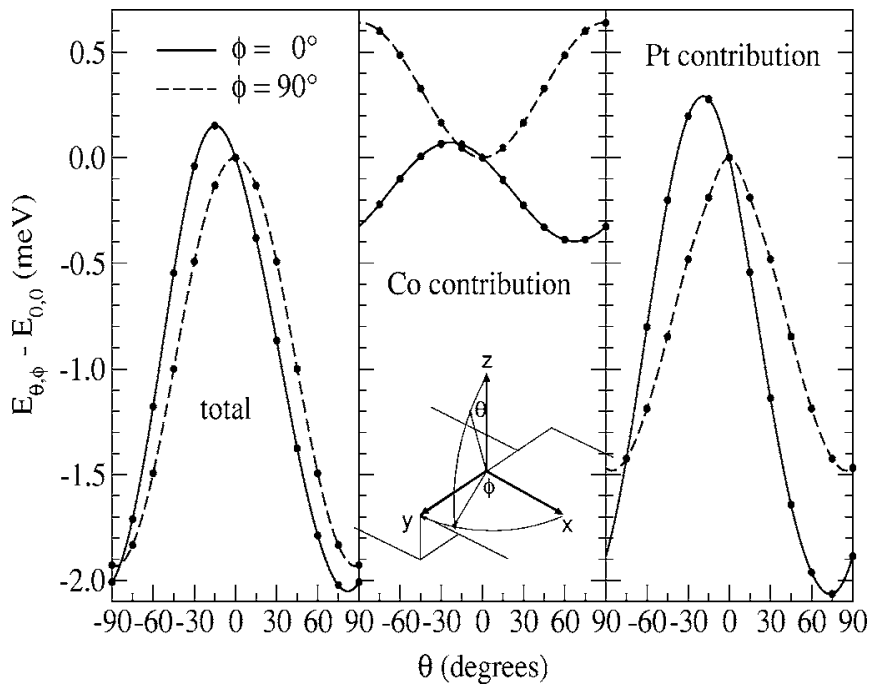

FIG. 6. The same as Fig. 4, but now for the relaxed system.
MAE. If we look at the direction of the easy axis, we see that it is now almost in the terrace plane $\left(\theta=71^{\circ}\right)$, and the "inplane" anisotropy, i.e., $E\left(\theta, \phi=90^{\circ}, 0^{\circ}\right)-E\left(\theta, \phi=90^{\circ}, 90^{\circ}\right)$, is very small. This effect seems to come mainly from the spin-orbit interaction in the substrate.

We have to conclude that in this case relaxation obviously worsens the agreement with experiment (at least, as far as the Co orbital moment and the direction of the easy axis are concerned). On the other hand, we know that with the inclusion of an orbital polarization scheme the orbital moment of Co can be enhanced. Since our above analysis has shown, that in the relaxed case the Co contribution to the magnetocrystalline anisotropy energy is only in the order of $15 \%-$ $20 \%$, the expected increase in the MAE as a result of the orbital polarization will be even smaller for the relaxed case than for the unrelaxed case.

\section{SUMMARY AND CONCLUSIONS}

Employing $a b$ initio calculations, we have demonstrated that relaxations on a Co decorated $\mathrm{Pt}$ step edge are in the order of $7 \%-13 \%$, while on a clean Pt step edge values of $2 \%-5 \%$ were obtained. Our calculations show that the structural inward relaxation of the step-edge atom is not necessarily visible in STM images, although we did not find an apparent outward relaxation for the clean Pt step edge, as found experimentally. The simulated STM images for Co/Pt(664) show an enhanced corrugation along the Co atoms, in agreement with experimental data. The influence of these relaxations on the magnetic properties of $\mathrm{Co} / \mathrm{Pt}(664)$ are (i) a pronounced decrease of the orbital moment of Co, (ii) a tilting of the easy axis towards the substrate, but (iii) the magnetic anisotropy (i.e., the energy difference between the easy and hard axes) remains almost unchanged. By decomposing the contributions of the Co and the Pt substrate to the MAE, we have shown that already in the unrelaxed geometry the substrate influence dominates, and this effect is even more pronounced in the relaxed system. We believe that relaxations will be an essential ingredient in calculations that in- 
clude orbital polarization schemes to determine both the orbital moment and the magnetic anisotropy, in reasonable agreement with experimental data. Although first promising calculations employing the LDA $+\mathrm{U}$ method to increase the orbital moment of Co have been made, ${ }^{8}$ these calculations require a self-consistent determination of the total energies for different magnetization directions and, thus, are computationally more demanding. Moreover, it is not trivial to de- termine the value of the Hubbard U, even in smaller systems. A parameter-free calculation scheme of the orbital polarization in the framework of the GW method has been proposed recently by Solovyev, ${ }^{28}$ and was successfully applied to bulk systems. Such calculations-with improved structural models-will be a challenge for future work on lowdimensional magnetic systems.
*Electronic address: G.Bihlmayer@fz-juelich.de

${ }^{1}$ Ultrathin Magnetic Structures, edited by J. A. C. Bland and B. Heinrich, (Springer, Berlin, 1994).

${ }^{2}$ P. Gambardella, A. Dallmeyer, K. Maiti, M. C. Malagoli, S. Rusponi, P. Ohresser, W. Eberhardt, C. Carbone, and K. Kern, Phys. Rev. Lett. 93, 077203 (2004).

${ }^{3}$ P. Gambardella, S. Rusponi, M. Veronese, S. S. Dhesi, C. Grazioli, A. Dallmeyer, I. Cabria, R. Zeller, P. H. Dederichs, and K. Kern, Science 300, 1130 (2003).

${ }^{4}$ P. Gambardella, A. Dallmeyer, K. Maiti, M. C. Malagoli, W. Eberhardt, K. Kern, and C. Carbone, Nature (London) 416, 301 (2002).

${ }^{5}$ A. J. Freeman and C. L. Fu, J. Appl. Phys. 67, 3356 (1987).

${ }^{6}$ A. J. Freeman and R.-Q. Wu, J. Magn. Magn. Mater. 100, 497 (1991).

${ }^{7}$ S. Blügel, B. Drittler, R. Zeller, and P. H. Dederichs, Appl. Phys. A: Solids Surf. 49, 547 (1989).

${ }^{8}$ A. B. Shick, F. Maca, and P. M. Oppeneer, Phys. Rev. B 69, 212410 (2004).

${ }^{9}$ B. Újfalussy, B. Lazarovits, L. Szunyogh, G. M. Stocks, and P. Weinberger, Phys. Rev. B 70, 100404(R) (2004).

${ }^{10}$ E. Wimmer, H. Krakauer, M. Weinert, and A. J. Freeman, Phys. Rev. B 24, 864 (1981).

${ }^{11}$ For program description see http://www.flapw.de.

${ }^{12}$ J. P. Perdew, J. A. Chevary, S. H. Vosko, K. A. Jackson, M. R. Pederson, D. J. Singh, and C. Fiolhais, Phys. Rev. B 46, 6671 (1992).

${ }^{13}$ C. Li, A. J. Freeman, H. J. F. Jansen, and C. L. Fu, Phys. Rev. B 42, 5433 (1990).

${ }^{14}$ R. Yu, D. Singh, and H. Krakauer, Phys. Rev. B 43, 6411 (1991).
${ }^{15}$ G. Boisvert, L. J. Lewis, and M. Scheffler, Phys. Rev. B 57, 1881 (1998).

${ }^{16}$ P. J. Feibelman, Phys. Rev. B 52, 16845 (1995).

${ }^{17}$ T. Michely, M. Hohage, M. Bott, and G. Comsa, Phys. Rev. Lett. 70, 3943 (1993).

${ }^{18}$ P. Steadman, K. F. Peters, H. Isern, and S. Ferrer, Phys. Rev. B 64, 125418 (2001).

${ }^{19}$ S. Baud, C. Ramseyer, G. Bihlmayer, S. Blügel, C. Barreteau, M. C. Desjonquères, D. Spanjaard, and N. Bernstein, Phys. Rev. B 70, 235423 (2004).

${ }^{20}$ N. Materer, U. Starke, A. Barbieri, R. Doll, K. Heinz, M. A. V. Hove, and G. A. Somorjai, Surf. Sci. 325, 207 (1995).

${ }^{21}$ P. Gambardella, M. Blanc, H. Brune, K. Kuhnke, and K. Kern, Phys. Rev. B 61, 2254 (2000).

${ }^{22}$ J. Tersoff and D. R. Hamann, Phys. Rev. B 31, 805 (1985).

${ }^{23}$ S. Heinze, S. Blügel, R. Pascal, M. Bode, and R. Wiesendanger, Phys. Rev. B 58, 16432 (1998).

${ }^{24}$ M. Komelj, C. Ederer, J. W. Davenport, and M. Fähnle, Phys. Rev. B 66, 140407(R) (2002).

${ }^{25}$ B. Nonas, I. Cabria, R. Zeller, P. H. Dederichs, T. Huhne, and H. Ebert, Phys. Rev. Lett. 86, 2146 (2001).

${ }^{26}$ Using LDA + U for the calculation of the bulk CoPt alloy, A. B. Shick and O. N. Mryasov, Phys. Rev. B 67, 172407 (2003), demonstrated that inclusion of a Hubbard $\mathrm{U}$ also on the Pt sites can compensate the overestimation of the MAE and leads to a correct description of both the MAE and the Co orbital moment.

${ }^{27}$ I. V. Solovyev, P. H. Dederichs, and I. Mertig, Phys. Rev. B 52, 13419 (1996).

${ }^{28}$ I. V. Solovyev, Phys. Rev. Lett. 95, 267205 (2005). 